'Departamento de

Medicina Intensiva.

${ }^{2}$ Departamento de

Medicina Interna.

Facultad de Medicina,

Pontificia Universidad

Católica de Chile. Santiago

$$
\text { de Chile. }
$$

Recibido el 29 de marzo de 2012, aceptado el 19 de julio de 2012.

Correspondencia a:

Dr. Max Andresen $\mathrm{H}$.

Unidad de Paciente Crítico

Hospital Clínico Pontificia

Universidad Católica de

Chile.

Marcoleta $367,4^{\circ}$ piso.

Santiago, Chile.

Tel: 02-3543265

Fax: 02-3543703

E-mail: andresen@med.

puc.cl

\section{Controversias en tromboembolismo pulmonar masivo}

\author{
MARCELO MERCADO ${ }^{1}$, ANDRÉS AIZMAN ${ }^{2}$, MAX ANDRESEN ${ }^{1}$ MSC
}

\section{Controversies in massive pulmonary embolism}

Massive pulmonary embolism (PE) is associated with high mortality. There is still a broad assortment of severity classifications for patients with $P E$, which affects the choice of therapies to use. The main clinical criteria for defining a PE as massive is systemic arterial hypotension, which depends on the extent of vascular obstruction and the previous cardiopulmonary status. Right ventricular dysfunction is an important pathogenic element to define the severity of patients and short term clinical prognosis. The recommended treatment is systemic thrombolysis, but in centers with experience and resources, radiological invasive therapies through catheters are useful alternatives that can be used as first choice tools in certain cases.

(Rev Med Chile 2013; 141: 486-494).

Key words: Pulmonary embolism; Thrombectomy; Thrombolytic therapy.
$\mathrm{P}$ or largo tiempo la literatura médica se ha referido de distinta manera al tromboembolismo pulmonar (TEP) que amenaza la vida. Diagnósticos como TEP mayor ${ }^{1}$, TEP anatómicamente masivo, TEP radiológicamente masivo y TEP angiográficamente masivo ${ }^{2}$, TEP grave y TEP masivo ${ }^{3}$ son utilizados frecuente y a veces indistintamente al referirse a este tipo de pacientes. Esta variabilidad en las definiciones puede llegar a ser un obstáculo en la comprensión de la patología, contribuyendo a crear confusión en el clínico y dificultando la interpretación de las publicaciones sobre el tema.

Durante algún tiempo, la condición de gravedad del TEP se asoció a la magnitud del compromiso anatómico. De esta manera, el TEP anatómica o radiológicamente masivo (obstrucción mayor de $50 \%$ de los vasos pulmonares o de dos o más arterias lobares) ${ }^{2,4}$ se asumió como un cuadro de similar gravedad al TEP con compromiso hemodinámico.

La gravedad de los pacientes con TEP depende de la clínica y no exclusivamente de la extensión de compromiso radiológico o anatómico. El principal criterio para calificar un TEP como masivo es la hipotensión arterial sistemica ${ }^{5}$, es decir, un paciente con TEP masivo radiológico o angiográ- fico no necesariamente cursa con compromiso hemodinámico. El impacto clínico y los resultados dependen no solamente del tamaño del émbolo o de la carga embólica, sino también del estado cardiopulmonar basal del paciente.

En los casos fatales, la mayoría de las muertes se producen dentro de la primera hora de la presentación clínica y los TEP anatómicamente masivos son responsables de solamente la mitad de estos casos ${ }^{6}$.

El TEP tiene una mortalidad y morbilidad asociada que se mantiene elevada a pesar de avances en terapia y diagnóstico. La mortalidad varía ampliamente dependiendo de la gravedad clínica de la embolia y está en rangos de menos de 1\% hasta más de $50 \%$ en los casos más graves ${ }^{7-11}$. El TEP masivo está asociado a un peor pronóstico y a tasas de mortalidad de hasta $65 \%$ en la fase aguda $^{12}$. La mayoría de las muertes secundarias al TEP se deben a falla cardiaca refractaria y no a insuficiencia respiratoria.

La presencia de disfunción o falla ventricular derecha secundaria a una sobrecarga aguda de presión, es el elemento fisiopatológico más importante para definir la gravedad de los pacientes y el pronóstico clínico a corto plazo ${ }^{13}$.

El grado de disfunción ventricular derecha 
esta directamente relacionado con el tamaño del émbolo y la magnitud de la obstrucción vascular, sin embargo, existen otros elementos que se deben tomar en cuenta, como la disfunción miocárdica persistente, vasoconstricción pulmonar, grado de activación de las plaquetas $y$, fundamentalmente, enfermedades cardiacas o pulmonares preexistentes que pueden exagerar el impacto de la embolia ${ }^{14-17}$.

La Sociedad Europea de Cardiología, en las guías publicadas en el año 2008, sugieren el reemplazo de las definiciones de TEP utilizadas actualmente: "no masivo, submasivo y masivo" por las de "TEP de alto riesgo" y "no alto riesgo" (que incluye TEP no masivo y submasivo). Según esta clasificación, el TEP de alto riesgo incluye los cuadros más graves (hipotensión y shock) que reúnen aproximadamente $5 \%$ de todos los casos de TEP y se asocia a un elevado riesgo de mortalidad intrahospitalaria, principalmente dentro de las primeras horas de admisión ${ }^{18}$.

El TEP masivo se define como el TEP agudo con hipotensión sostenida (PAS $<90 \mathrm{mmHg}$ ) durante al menos $15 \mathrm{~min}$ o que requiera soporte inotrópico, que no sea secundario a otra causa además del TEP, como arritmias, hipovolemia, sepsis o disfunción ventricular izquierda, ausencia de pulso o bradicardia profunda persistente (frecuencia cardiaca $<40 \mathrm{lpm}$ con signos y síntomas de shock) $)^{3}$.

\section{Consideraciones terapéuticas}

Los pacientes con TEP masivo requieren una permeabilizacion inmediata, ya sea farmacológica o mecánica de las arterias pulmonares ocluidas $^{3,18,19}$.

Las guías y consensos publicados recientemente, todavía favorecen la utilización de la trombolisis sistémica en el TEP masivo, (recomendación 2C) por sobre la trombectomía mecánica o embolectomía quirúrgica, herramientas igualmente útiles en caso de existir contraindicación absoluta y dependiendo de la experiencia del centro ${ }^{3,18,19}$.

\section{Trombolisis farmacológica}

El efecto de los trombolíticos en el TEP ha sido evaluado en varios estudios controlados $(\mathrm{RCT})^{3,19,20}$, sin embargo, solamente en 5 RCT se incluyeron pacientes con TEP masivo entre los sujetos estudiados. Sólo un estudio de estos últimos ${ }^{21}$ estudió en forma exclusiva pacientes con TEP masivo, mostrando que 4/4 pacientes que recibieron terapia trombolítica sobrevivieron vs 4/0 de los que recibieron anticoagulación.

Los trombolíticos favorecen la lisis de las moléculas de fibrina, a diferencia de la heparina que solamente produce una reducción pasiva del tamaño del trombo.

Algunos estudios han mostrado en forma clara una rápida resolución de la obstrucción tromboembólica con la administración de trombolíticos, logrando de esta forma una mejoría más rápida de los parámetros hemodinámicos (elevación significativa del gasto cardiaco, disminución de la presión sistólica y media de arteria pulmonar) que con la administración de heparina sola ${ }^{22,23}$.

Existen riesgos potenciales al utilizar trombolíticos, pero los beneficios son claramente mayores, y entre estos se incluyen: la rápida resolución de los síntomas, estabilización de la función respiratoria y cardiovascular, disminución de la necesidad de soporte ventilatorio y de drogas vasopresoras, reducción del daño al ventrículo derecho, disminución de la recurrencia y un incremento en la posibilidad de sobrevida ${ }^{3}$. Los riesgos incluyen sangrados o hemorragias fatales, hemorragias intracerebrales y sangrados menores que pueden prolongar la hospitalización y requerir soporte transfusional.

Para el tratamiento del TEP masivo, se han utilizado diversos esquemas de trombolíticos (Tabla 1).

Cuando se utiliza trombolisis, se sugieren períodos cortos de infusión (por ej. rt-PA en dos horas) por sobre las infusiones prolongadas (recomendación 2C), sin embargo, no se demostraron diferencias en el resultado hemodinámico al final de las infusiones prolongadas ${ }^{19,23,24}$.

Todos los esquemas parecen ser al menos comparables en términos de eficacia, sin embargo, debería privilegiarse el uso de rt-PA por sobre las infusiones prolongadas de estreptoquinasa $y$ uroquinasa.

El beneficio hemodinámico que se alcanza con los trombolíticos a corto plazo, parece ser evidente solamente en los primeros días. Al finalizar la primera semana, no se encontraron diferencias entre los pacientes tratados con heparina vs alteplasa, en relación a modificaciones en el grado de obstruc- 
Tabla 1. Esquemas de trombolisis sistémica

\begin{tabular}{|llc|}
\hline Droga & Dosis & $\begin{array}{c}\text { Aprobado por FDA para } \\
\text { el tratamiento de TEP }\end{array}$ \\
Estreptokinasa (SK) & $\begin{array}{l}250.000 \mathrm{UI} \text { carga en } 30 \mathrm{~min} \text {, seguidos de } 100.000 \mathrm{UI} \text { hora en } 12-24 \mathrm{~h} \\
\text { Esquema acelerado: } 1,5 \text { millones UI en } 2 \mathrm{~h}\end{array}$ & Sí \\
Urokinasa (UK) & $\begin{array}{l}4.400 \mathrm{UI} / \mathrm{kg} / \mathrm{h} \text { en } 12-24 \mathrm{~h} \\
\text { Esquema acelerado: } 3 \text { millones de UI en } 2 \mathrm{~h}\end{array}$ \\
rt-PA (Alteplasa) & $\begin{array}{l}100 \mathrm{mg} \text { en } 2 \mathrm{~h} \\
0,6 \mathrm{mg} / \mathrm{kg} \text { en } 15 \mathrm{~min} \text { (dosis máxima } 50 \mathrm{mg})\end{array}$ & sí \\
Reteplase & Dos bolos de $10 \mathrm{U}$ ev separados por $10 \mathrm{~min}$ & No \\
\hline
\end{tabular}

ción vascular y en las mediciones ecocardiográficas realizadas ${ }^{25}$.

$\mathrm{Wan}^{20}$, al analizar los pacientes con TEP masivo, logró identificar una reducción de la recurrencia de TEP o muerte de 19,0\% con heparina sola a $9,4 \%$ con trombolisis (OR $0,45,95 \%$ CI 0,22 a $0,90)$. El número necesario para tratar para prevenir el "end point" combinado de TEP recurrente o muerte fue de 10 , no se demostró un incremento significativo en los sangrados mayores pero si un incremento en los sangrados no mayores, el número necesario para dañar fue de 8 .

El riesgo de hemorragia asociado a la trombolisis existe y es mayor en relación a las comorbilidades que presenta el paciente. El riesgo en datos acumulados de RCTs muestra 13\% de sangrado mayor y $1,8 \%$ de hemorragia intracraneal o hemorragia fatal ${ }^{26-31}$. El riesgo de un sangrado mayor después de la trombolisis puede ser de hasta 4,2\% cuando se realiza un diagnóstico no invasivo y hasta $14 \%$ después de un diagnóstico invasivo de $\mathrm{TEP}^{32}$.

Resulta difícil establecer contraindicaciones para la trombolisis en el TEP dado el escaso número de pacientes estudiados en los RCT publicados, en los que se comparan trombolisis vs placebo ${ }^{3,20}$. Por lo tanto, las contraindicaciones para la trombolisis suelen ser extrapoladas de datos obtenidos de estudios en pacientes con IAM con SDST ${ }^{33,34}$ (Tabla 2).

Al evaluar los posibles riesgos vs los potenciales beneficios de la trombolisis, es importante tener en cuenta la historia natural y el pronóstico del TEP. De esta manera, la que sería una contraindicación absoluta de trombolisis en el infarto agudo del miocardio, puede ser solamente relativa en un paciente con TEP masivo potencialmente fatal ${ }^{18}$.
En resumen, la trombolisis es el tratamiento de primera línea en los pacientes con TEP que se presentan con clínica de shock cardiogénico o hipotensión arterial persistente. El uso de trombolisis debe ser considerado en forma individual en los pacientes con riesgo intermedio y no debe utilizarse en pacientes de bajo riesgo (Tabla 3 ).

\section{Terapias realizadas mediante catéteres}

Los objetivos de la terapia realizada por catéter son: producir una rápida reducción de la presión de arteria pulmonar, de la sobrecarga del VD y de

\section{Tabla 2. Contraindicaciones para la terapia trombolítica}

\section{Contraindicaciones absolutas}

- AVE hemorrágico o AVE de origen desconocido en cualquier momento

- AVE isquémico los seis meses previos

- Sospecha de disección aortica

- Sangramiento activo o diátesis hemorrágica

- Trauma mayor/cirugía/TEC mayor reciente (3 semanas previas)

- Sangramiento gastrointestinal el último mes

\section{Contraindicaciones relativas}

- Edad > 75 años

- Embarazo

- Punciones vasculares no compresibles

- RCP traumática o prolongada (> $10 \mathrm{~min}$ )

- TIA en los 6 meses previos

- Hipertensión refractaria (presión sistólica > $180 \mathrm{mmHg}$ )

- Tratamiento anticoagulante oral

- Enfermedad hepática avanzada

- Endocarditis infecciosa

- Úlcera péptica activa 
Tabla 3. Resumen de grados de recomendación para el tratamiento del TEP masivo (GRADE), Guías de práctica clínica ACCP, 9a 19

Condición clínica
Trombolisis sistémica en pacientes con hipotensión
Trombolisis en pacientes con TEP sin hipotensión, bajo
riesgo de sangramiento y alto riesgo de desarrollar
hipotensión
Cuando se decide el uso de trombolisis
Cuando se decide el uso de trombolisis

En pacientes con TEP e hipotensión que tienen: contraindicación para trombolisis, falla en la trombolisis o shock que puede causar la muerte antes de que la trombolisis pueda ser efectiva

En pacientes con TEP e hipotensión que tienen: contraindicación para trombolisis, falla en la trombolisis o shock que puede causar la muerte antes de que la trombolisis pueda ser efectiva

\section{Recomendación}

Se sugiere trombolisis

$2 \mathrm{C}$

Se sugiere la administración de trombolisis

Se sugieren infusiones cortas por sobre las prolongadas

$2 C$

Se sugiere la administración a través de una vena perifé- $\quad 2 C$ rica por sobre un catéter en arteria pulmonar

Se sugiere la terapia asistida por catéter, si existen la $2 C$ experiencia y los recursos adecuados

Se sugiere la embolectomía quirúrgica, si la experiencia $2 C$ quirúrgica y los recursos están disponibles

Grado 2C: Recomendación débil, evidencia de baja o muy baja calidad. Incertidumbre en la estimación de riesgo/beneficio. Otras alternativas podrían ser igualmente razonables.

la hipertensión pulmonar, mejorar la perfusión sistémica y facilitar la recuperación del VD.

En nuestro centro, la experiencia en el diagnóstico y tratamiento del TEP a través de catéteres (fragmentación, trombolisis local) y monitorización de la reperfusión a través de biomarcadores ha sido satisfactoria, con adecuada respuesta clínica y angiográfica, no registrándose complicaciones hemorrágicas mayores ${ }^{35-38}$.

Las terapias realizadas por catéter son comparables a la trombolisis farmacológica sistémica en cuanto a efectividad y seguridad. Esta terapia puede realizarse como tratamiento de primera línea en centros seleccionados con experiencia en estos procedimientos y no solamente como terapia primaria en pacientes con contraindicación de trombolisis o como terapia de rescate, cuando la trombolisis falla en lograr mejoría hemodinámica en el cuadro agudo ${ }^{39,40}$.

Así, en ciertos pacientes con TEP masivo, la trombolisis no puede ser realizada por diferentes motivos: a) contraindicación absoluta de trombolíticos; b) alto riesgo de hemorragia; c) la embolectomía quirúrgica no está disponible o está contraindicada y d) cuando por el estado de gravedad del paciente, no existe tiempo suficiente para esperar el efecto de la trombolisis.

En estos pacientes, las terapias realizadas por catéter son útiles para revertir la falla ventricular derecha, sin la exposición a la trombolisis farmacológica ni a la circulación extracorpórea en el caso de la embolectomía quirúrgica ${ }^{19}$. Estas terapias se pueden implementar rápidamente y con más facilidad que la embolectomía quirúrgica.

Existen tres tipos de intervenciones que se pueden realizar a través del catéter:

a) Aspiración de los trombos: la aspiración sostenida en la punta del catéter permite sostener el embolo mientras es removido habitualmente por una venotomía.

b) Fragmentación: se realiza por catéteres simples de angiografía ("pigtail"), guías, balones de angioplastía o por catéteres más avanzados como el catéter de Amplatz (Microvena Corp.) que utiliza un impulsor para homogeneizar el trombo.

c) Trombectomía reolítica: a través de catéteres como el Angiojet (Possis Medical, Inc.); Hydrolyser (Cordis Corp) y Oasis (Meditech/ Boston Scientific Corp.) que utilizan un "jet" de solución salina a alta velocidad para crear un efecto Venturi que fragmenta los trombos y luego los aspira por un lumen de evacuación ${ }^{41}$.

Ninguna de las técnicas ha probado ser mejor que otra. Este tipo de terapia debe realizarse 


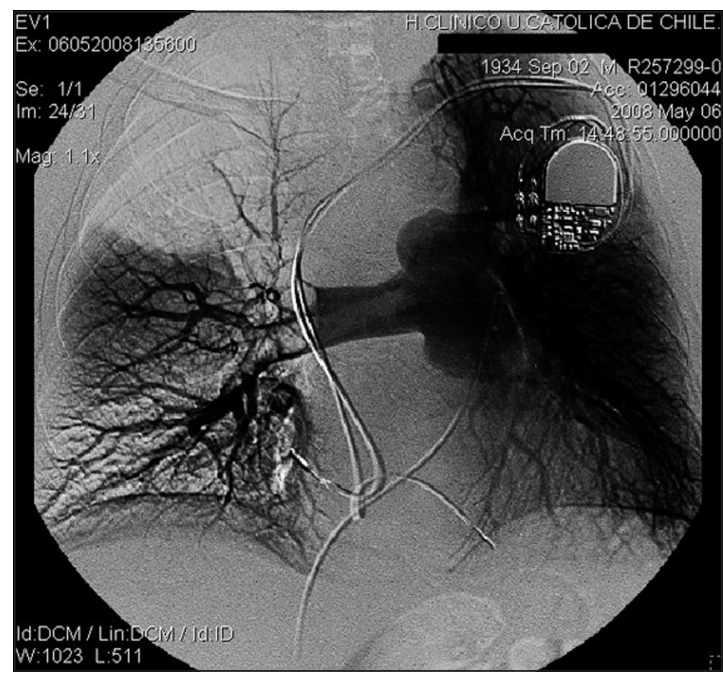

Figura 1. Pre trombolisis. Se observa gran defecto de llene en arteria pulmonar derecha.

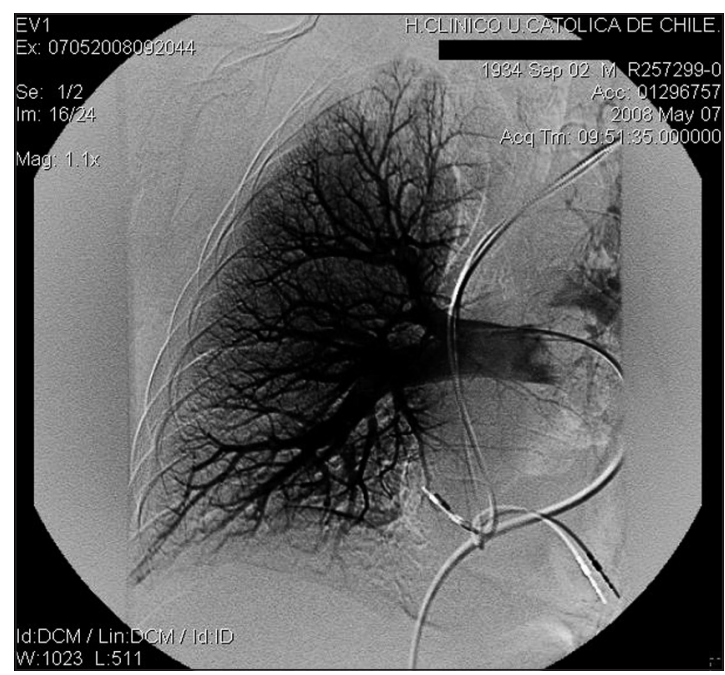

Figura 3. Control post trombolisis. Completa reperfusión de pulmón derecho.

solamente en las arterias principales, ya que el beneficio que se obtiene en las arterias de menor calibre es escaso y el riesgo de lesión y perforación es mayor ${ }^{3}$.

Las terapias híbridas son estrategias que se realizan en forma exitosa desde hace varios años, combinando la fragmentación mecánica del coágulo con la trombolisis farmacológica local en dosis más bajas que las utilizadas en forma sistémica, logrando una concentración más ele-

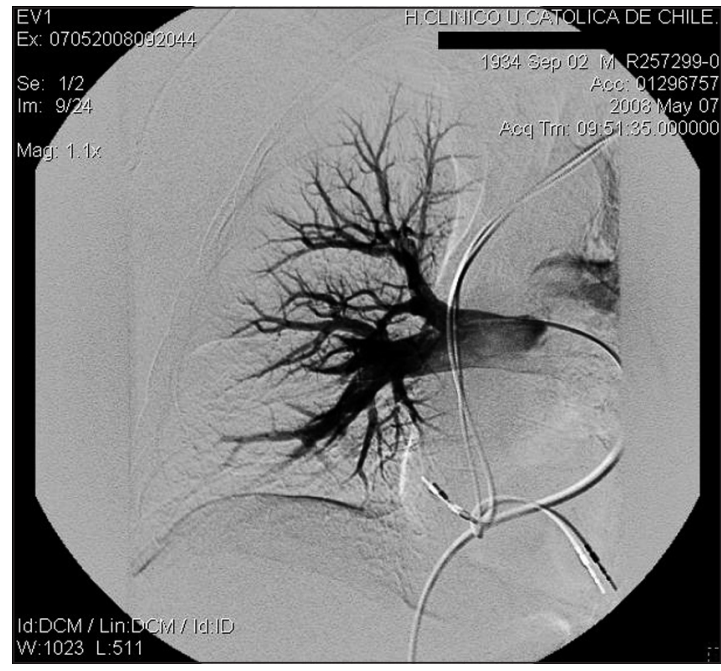

Figura 2. Catéter intra trombo en arteria pulmonar derecha, durante la trombolisis. Normalización de presión arterial.

vada del fármaco en el sitio de obstrucción, con eficacia mayor y menor riesgo de complicaciones hemorrágicas ${ }^{37,41,42}$ (Figura 1-3).

La fragmentación es la técnica más utilizada en los estudios reportados: en hasta $60 \%$ de los casos se utiliza en forma complementaria la trombolisis local $^{40,43}$.

El procedimiento debe ser finalizado tan pronto como se consiga mejoría hemodinámica, independientemente del resultado angiográfico, ya que cambios modestos en la angiografía pueden lograr una notable mejoría hemodinámica. Se puede lograr mejoría clínica relevante sin necesidad de obtener una completa repermeabilización, esto porque mejorías parciales del flujo pueden ser suficientes para incrementar el gasto cardiaco y revertir el compromiso hemodinámico ${ }^{41}$.

El calibre de las arterias pulmonares principales y la gran carga de trombos puede limitar la eficacia de la terapia, que se realiza muchas veces con instrumentos diseñados para vasos de menor calibre. El único catéter aprobado por la (Food and Drugs Administration (FDA) de los Estados Unidos de Norteamérica para el tratamiento del TEP es el de Greenfield, diseñado hace más de 50 años $^{44}$.

\section{Trombolisis por catéter}

Después de la fragmentación mecánica, una mayor superficie del trombo queda expuesta al 
trombolítico por lo que se necesita una menor dosis del fármaco para obtener lisis efectiva. Los datos acumulados en pequeños estudios muestran que la fragmentación puede lograr mejoría hemodinámica en hasta 95\% de los pacientes cuando se utilizan en conjunto con trombolíticos locales ${ }^{40}$.

La administración del agente trombolítico en la arteria pulmonar, directamente concentrado en el embolo, permite disminuir la dosis del fármaco y de esta manera reducir los eventuales efectos adversos, permitiendo acelerar la lisis de los coágulos y la reperfusión de las arterias pulmonares.

La técnica requiere posicionar el catéter junto o mejor dentro del trombo, inyección de un bolo de 200.000-500.000 UI de uroquinasa, fragmentación mecánica con catéter y finalmente con el catéter posicionado en el segmento arterial más obstruido, infusión de uroquinasa $100.000 \mathrm{IU} / \mathrm{h}$ por 12 a 24 $\mathrm{h}$, algunos autores mantienen anticoagulación con heparina en forma simultánea a la tromboli$\operatorname{sis}^{37,39,45}$. Otros esquemas utilizan $10 \mathrm{mg}$ de rt-PA seguido por una infusión de $20 \mathrm{mg} / \mathrm{h}$ en $2 \mathrm{~h}$.

\section{Complicaciones}

El metaanálisis de Kuo et $\mathrm{a}^{43}$ muestra que las terapias percutáneas pueden ser realizadas en forma segura, logran restablecer la estabilidad hemodinámica en $86,5 \%$ de los pacientes, con una tasa de sangrado mayor de $2,4 \%$.

Dentro de las complicaciones raras están las lesiones a la arteria pulmonar o sus ramas, taponamiento cardiaco, hemoptisis y deterioro hemodinámico secundario a embolización distal ${ }^{45}$.

Solamente el estudio de Verstraete et al. ${ }^{46}$ en pacientes con "TEP masivo agudo" (> 48\% de obstrucción vascular en angiografía) comparó la trombolisis sistémica vs la trombolisis intra arterial pulmonar con las mismas dosis de rt-PA. La infusión local no demostró un beneficio por sobre la vía sistémica. A partir de este estudio se establece la recomendación a favor de la trombolisis sistémica en la última guía del American College of Chest Physicians (ACCP ${ }^{19}$. Sin embargo, en este estudio no se incluyeron pacientes con compromiso hemodinámico, no se realizó ningún tipo de fragmentación, la administración de trombolíticos se realizó en la arteria pulmonar principal y no en contacto con el trombo.
El lograr una reducción de la obstrucción proximal sin una embolización distal excesiva, seguida de trombolisis local por catéter, parece ser la mejor estrategia.

En centros con la experiencia y los recursos apropiados, las terapias realizadas por catéter pueden ser la primera opción terapéutica en el TEP masivo.

La mejor evidencia disponible indica que la terapia por catéter moderna debería ser considerada también como una opción terapéutica de primera línea en pacientes con TEP masivo, en centros con experiencia adecuada.

Sin embargo, aún se requieren estudios prospectivos para confirmar los hallazgos del metaanálisis de Kuo, se necesitan además protocolos de manejo uniforme en los pacientes en los que se van a aplicar estas terapias y criterios bien definidos para evaluar los resultados y las posibles complicaciones.

\section{Embolectomía quirúrgica}

En pacientes con TEP masivo la embolectomía quirúrgica es una estrategia de rescate efectiva cuando hay contraindicación para la trombolisis, como terapia cuando la respuesta a la trombolisis es desfavorable, existe presencia de foramen oval permeable o trombo intracardiaco, además en presencia de shock que puede causar la muerte antes que la trombolisis pueda ser efectiva. Esta terapia debe estar reservada para centros con experiencia y recursos adecuados (Grado de recomendación 2C en la 9a. Ed. guía ACCP) $)^{19,47-49}$.

Durante mucho tiempo fue considerada solamente como una terapia de rescate por los pocos y desalentadores datos de su seguridad y eficacia, series antiguas registraban una mortalidad entre 20 y $30 \%{ }^{50}$. a) Existen reportes recientes de su uso en varios centros, con resultados prometedores que muestran mortalidad tan baja como 6-8\% a los 30 días, y sobrevida de $96 \%$ a los 4 años y de $83,5 \%$ a los 10 años ${ }^{18,48-51}$.

La trombolisis sistémica no es una contraindicación para la cirugía, sin embargo, pueden presentarse sangrados mayores a los habituales ${ }^{52}$.

No existen RCT que comparen esta técnica vs trombolisis o terapias por catéter, por lo que su uso, por ahora, debería seguir restringido a un grupo seleccionado de pacientes. 


\section{Filtro de vena cava inferior}

En el TEP los filtros de vena cava inferior (FVCI) pueden ser utilizados cuando existe contraindicación para anticoagulación por un riesgo significativo de hemorragia (recomendación 1B) o como terapia coadyuvante al tratamiento anticoagulante en pacientes seleccionados ${ }^{19}$.

No existe certeza acerca del beneficio de los FVCI en los pacientes con TEP masivo como terapia coadyuvante a la trombolisis y anticoagulación. En el registro ICOPER, la inserción de FVCI en los pacientes con TEP y compromiso hemodinámico se asocio a reducción de recurrencia precoz y de muerte $^{6}$.

La recomendación contra la inserción de FVCI en los pacientes con TEP masivo no se aplica al grupo de pacientes con compromiso hemodinámico $^{19}$.

Así, una adecuada clasificación y estratificación del riesgo nos permite en ciertos casos el empleo de terapia trombolítica, siempre evaluando riesgo vs beneficio; pensamos que el desarrollo y creciente empleo de variadas técnicas de radiología intervencional permitirá en breve plazo definir su preciso rol en el manejo de esta severa complicación.

\section{Referencias}

1. Hirsh J, Hoak J. Management of deep vein thrombosis and pulmonary embolism. A statement for healthcare professionals. Council on Thrombosis (in consultation with the Council on Cardiovascular Radiology), American Heart Association. Circulation 1996; 93: 2212-45.

2. Urokinase Pulmonary Embolism Trial. Phase 1 results: a cooperative study. JAMA 1970; 214: 2163-72.

3. Jaff M, McMurtry M, Archer S, Cushman M, Goldenberg N, Goldhaber S, et al. Management of Massive and Submassive Pulmonary Embolism, Iliofemoral Deep Vein Thrombosis, and Chronic Thromboembolic Pulmonary Hypertension. A Scientific Statement from the American Heart Association. Circulation 2011; 123: 1788-830.

4. Dalen J, Banas J Jr, Brooks HL, Evans GL, Paraskos JA, Dexter L. Resolution rate of acute pulmonary embolism in man. N Engl J Med 1969; 280: 1194-7.

5. Goldhaber SZ. Pulmonary embolism. Lancet 2004; 363: 1295-305.

6. Wood K. Major Pulmonary Embolism: Review of a Pathophysiologic Approach to the Golden Hour of Hemodynamically Significant Pulmonary Embolism. Chest 2002; 121: 877-905.
7. Goldhaber SZ, Visani L, De Rosa M. Acute pulmonary embolism: clinical outcomes in the International Cooperative Pulmonary Embolism Registry (ICOPER). Lancet 1999; 353: 1386-9.

8. Carson JL, Kelley MA, Duff A, Weg JG, Fulkerson WJ, Palevsky HI et al. The clinical course of pulmonary embolism. N Engl J Med 1992; 326: 1240-5.

9. Aujesky D, Jiménez D, Mor MK, Geng M, Fine MJ, Ibrahim SA. Weekend versus weekday admission and mortality after acute pulmonary embolism. Circulation 2009; 119: 962-8.

10. Laporte S, Mismetti P, Decousus H, Uresandi F, Otero R, Lobo J, Monreal M. Clinical predictors for fatal pulmonary embolism in 15, 520 patients with venous thromboembolism: findings from the Registro Informatizado de la Enfermedad Trombo Embolica Venosa (RIETE Registry). Circulation 2008; 117: 1711-6.

11. Kasper W, Konstantinides S, Geibel A, Tiede N, Krause T, Just H. Prognostic significance of right ventricular afterload stress detected by echocardiography in patients with clinically suspected pulmonary embolism. Heart 1997; 77: 346-9.

12. Kasper W, Konstantinides S, Geibel A, Olschewski M, Heinrich F, Grosser KD, et al. Management strategies and determinants of outcome in acute major pulmonary embolism: results of a multicenter registry. J Am Coll Cardiol 1997; 30: 1165-71.

13. Konstantinides S. Pulmonary embolism: impact of right ventricular dysfunction. Curr Opin Cardiol 2005; 20 : 496-501.

14. McIntyre K, Sasahara AA. Determinants of right ventricular function and hemodynamics after pulmonary embolism. Chest 1974; 65: 534-43.

15. Greyson C, Xu Y, Cohen J, Schwartz GG. Right ventricular dysfunction persists following brief right ventricular pressure overload. Cardiovasc Res 1997; 34: 281-8.

16. Schmitto JD, Doerge H, Post H, Coulibaly M, Sellin C, Popov AF. Progressive right ventricular failure is not explained by myocardial ischemia in a pig model of right ventricular pressure overload. Eur J Cardiothorac Surg 2009; 35: 229-34.

17. Chung T, Connor D, Joseph J, Emmett L, Mansberg R, Peters $\mathrm{M}$, et al. Platelet activation in acute pulmonary embolism. J Thromb Haemost 2007; 5: 918-24.

18. Torbicki A, Perrier A, Konstantinides S, Agnelli G, Galiè N, Pruszczyk P, et al. Guidelines on the diagnosis and management of acute pulmonary embolism: The Task Force for the Diagnosis and Management of Acute Pulmonary Embolism of the European Society of Cardiology (ESC). Eur Heart J 2008; 29: 2276-315.

19. Kearon C, Akl E, Comerota A, Prandoni P, Bounameaux 
H, Goldhaber SZ, et al. Antithrombotic Therapy and Prevention of Thrombosis, 9th ed: American College of Chest Physicians. Evidence-Based Clinical Practice Guidelines. Chest 2012; 141 (2) (Suppl): e419S-94S.

20. Wan S, Quinlan D, Agnelli G, Eikelboom J. Thrombolysis compared with heparin for the initial treatment of pulmonary embolism: a metaanalysis of the randomized controlled trials. Circulation 2004; 110: 744-9.

21. Jerjes-Sánchez C, Ramírez-Rivera A, de Lourdes García M, Arriaga-Nava R, Valencia S, Rosado-Buzzo A, et al. Streptokinase and heparin versus heparin alone in massive pulmonary embolism: a randomized controlled trial. J Thromb Thrombolysis 1995; 2: 227-9.

22. Tibbutt D, Davies J, Anderson J, Fletcher EW, Hamill J, Holt JM, et al. Comparison by controlled clinical trial of streptokinase and heparin in treatment of lifethreatening pulmonary embolism. BMJ 1974; 1: 343-7.

23. Goldhaber S, Kessler C, Heit J, Markis J, Sharma GV, Dawley D, et al. Randomised controlled trial of recombinant tissue plasminogen activator versus urokinase in the treatment of acute pulmonary embolism. Lancet 1988; 2: 293-8.

24. Meneveau N, Schiele F, Metz D, Valette B, Attali P, Vuillemenot A, et al. Comparative efficacy of a two-hour regimen of streptokinase versus alteplase in acute massive pulmonary embolism: immediate clinical and hemodynamic outcome and one-year follow-up. J Am Coll Cardiol 1998; 31: 1057-63.

25. Konstantinides S, Tiede N, Geibel A, Olschewski M, Just $\mathrm{H}$, Kasper W. Comparison of alteplase versus heparin for resolution of major pulmonary embolism. Am J Cardiol 1998; 82: 966-90.

26. The urokinase pulmonary embolism trial. A national cooperative study. Circulation 1973; 47 (2 Suppl. II): 1-108.

27. Dalla-Volta S, Palla A, Santolicandro A, Giuntini C, Pengo V, Visioli O, et al. PAIMS 2: alteplase combined with heparin versus heparin in the treatment of acute pulmonary embolism. Plasminogen activator Italian multicenter study 2 J Am Coll Cardiol 1992; 20: 520-6.

28. Levine M, Hirsh J, Weitz J, Cruickshank M, Neemeh J, Turpie AG, et al. A randomized trial of a single bolus dosage regimen of recombinant tissue plasminogen activator in patients with acute pulmonary embolism. Chest 1990; 98: 1473-9.

29. Konstantinides S, Geibel A, Heusel G, Heinrich F, Kasper W; Management Strategies and Prognosis of Pulmonary Embolism-3 Trial Investigators. Heparin plus alteplase compared with heparin alone in patients with submassive pulmonary embolism. N Engl J Med 2002; 347: 1143-50.
30. Kanter D, Mikkola K, Patel S, Parker JA, Goldhaber SZ. Thrombolytic therapy for pulmonary embolism. Frequency of intracranial hemorrhage and associated risk factors. Chest 1997; 111: 1241-5.

31. Goldhabert S, Kessler C, Heit J, Elliott CG, Friedenberg W, Heiselman D, et al. Recombinant tissue-type plasminogen activator versus a novel dosing regimen of urokinase in acute pulmonary embolism: a randomized controlled multicenter trial. J Am Coll Cardiol 1992; 20: 24-30.

32. Stein P, Hull R, Raskob G. Risks for major bleeding from thrombolytic therapy in patients with acute pulmonary embolism. Ann Intern Med 1994; 121: 313-7.

33. Antman E, Anbe D, Armstrong P, Bates ER, Green LA, Hand $\mathrm{M}$, et al. ACC/AHA guidelines for the management of patients with ST-elevation myocardial infarction: a report of the American College of Cardiology/ American Heart Association Task Force on Practice Guidelines (Committee to Revise the 1999 Guidelines for the Management of Patients with Acute Myocardial Infarction)]. Circulation 2004; 110: e82-e292.

34. Van de W, Ardissino D, Betriu A, Cokkinos DV, Falk E, Fox KA, et al. Management of acute myocardial infarction in patients presenting with ST-segment elevation. The Task Force on the Management of Acute Myocardial Infarction of the European Society of Cardiology. Eur Heart J 2003; 24: 28-66.

35. Andresen M, González A, Díaz O, Meneses L, Fava M, Orlandini E, et al. Diagnostic yield of CT pulmonary angiography and venography for thromboembolic disease. Rev Med Chile 2010; 138: 1480-6.

36. Andresen M, González A, Mercado M, Díaz O, Meneses L, Fava M, et al. Natriuretic peptide type-B can be a marker of reperfusion in patients with pulmonary thromboembolism subjected to invasive treatment. Int J Cardiovasc Imaging. 2011.

37. Fava M, Loyola S, Meneses L. [Pulmonary embolism treated with pharmacological thrombolysis and mechanical fragmentation. Report of one case]. Rev Med Chile 2005; 133: 1477-82.

38. Fava M, Loyola S, Bertoni H, Dougnac A. Massive pulmonary embolism: percutaneous mechanical thrombectomy during cardiopulmonary resuscitation. Vasc Interv Radiol 2005; 16: 119-23.

39. de Gregorio M, Laborda A, de Blas I, Medrano J, Mainar A, Oribe M. Endovascular Treatment of a Haemodynamically Unstable Massive Pulmonary Embolism using Fibrinolysis and Fragmentation. Experience with 111 Patients in a Single Centre. Why don't we follow ACCP Recomendations? Arch Bronconeumol 2011; 47: 17-24. 
40. Skaf E, Beemath A, Siddiqui T, Janjua M, Patel NR, Stein PD. Catheter-tip embolectomy inthe management of acute massive pulmonary embolism. Am J Cardiol 2007; 99: 415-20.

41. Teodoran T, Sobieszczyk P. Catheter-Based Therapies for Massive Pulmonary Embolism. Prog Cardiovasc Dis 2010; 52: 429-37.

42. Fava M, Loyola S. Applications of percutaneous mechanical thrombectomy in pulmonary embolism. Tech Vasc Interv Radiol. 2003; 6: 53-8.

43. Kuo W, Gould M, Louie J, Rosenberg JK, Sze DY, Hofmann LV. Catheter-directed therapy for the treatment of massive pulmonary embolism: Systematic review and meta-analysis of modern techniques. J Vasc Interv Radiol 2009; 20: 1431-40.

44. Greenfield L, Kimmell G, McCurdy III W. Transvenous removal of pulmonary emboli by vacuum-cup catheter technique. J Surg Res 1969; 9: 347-52.

45. Nakazawa K, Tajima H, Murata S, Kumita SI, Yamamoto $\mathrm{T}$, Tanaka K. Catheter fragmentation of acute massive pulmonary thromboembolism: distal embolisation and pulmonary arterial pressure elevation. Br J Radiol 2008; 81: 848-54.

46. Verstraete M, Miller GA, Bounameaux H, Charbonnier B, Colle JP, Lecorf G. Intravenous and intrapulmonary recombinant tissue-type plasminogen activator in the treatment of acute massive pulmonary embolism. Circulation 1988, 77: 353-60.

47. Meneveau N, Se'ronde M, Blonde, Legalery P, DidierPetit K, Briand F, et al. Management of unsuccessful thrombolysis in acute massive pulmonary embolism. Chest 2006; 129: 1043-50.

48. Yalamanchili K, Fleisher A, Lehrman S, Axelrod HI, Lafaro RJ, Sarabu MR, et al. Open pulmonary embolectomy for treatment of major pulmonary embolism. Ann Thorac Surg 2004; 77: 819-23.

49. Leacche M, Unic D, Goldhaber S, Rawn JD, Aranki SF, Couper GS, et al. Modern surgical treatment of massive pulmonary embolism: results in 47 consecutive patients after rapid diagnosis and aggressive surgical approach. J Thorac Cardiovasc Surg 2005; 129: 1018-23.

50. Stein P, Alnas M, Beemath A, Patel NR. Outcome of pulmonary embolectomy. Am J Cardiol 2007; 99: 421-3.

51. Fukuda I, Taniguchi S, Fukui K, Minakawa M, Daitoku K, Suzuki Y. Improved Outcome of Surgical Pulmonary Embolectomy by Aggressive Intervention for Critically Ill Patients. AnnThorac Surg 2011; 91: 728-32.

52. Aklog L, Williams C, Byrne J, Goldhaber S. Acute pulmonary embolectomy: a contemporary approach. Circulation 2002; 105: 1416-9. 